Short Communication

\title{
Effect of combination therapy containing a high-dose carbapenem on mortality in patients with carbapenem-resistant Klebsiella pneumoniae bloodstream infection
}

\author{
Maddalena Giannella ${ }^{\text {a,* }}$, Enrico Maria Trecarichi ${ }^{\text {b }}$, Daniele Roberto Giacobbe ${ }^{\text {, }}$ \\ Francesco Giuseppe De Rosa ${ }^{\mathrm{d}}$, Matteo Bassetti ${ }^{\mathrm{e}}$, Alessandro Bartoloni ${ }^{\mathrm{f}}$, \\ Michele Bartoletti ${ }^{\mathrm{a}}$, Angela Raffaella Losito ${ }^{\mathrm{b}}$, Valerio del Bono ${ }^{\mathrm{c}}$, Silvia Corcione ${ }^{\mathrm{d}}$, \\ Sara Tedeschi ${ }^{\text {a }}$, Francesca Raffaelli ${ }^{\text {b }}$, Carolina Saffioti ${ }^{c}$, Teresa Spanu ${ }^{g}$, \\ Gian Maria Rossolini ${ }^{\mathrm{h}}$, Anna Marchese ${ }^{\mathrm{i}}$, Simone Ambretti ${ }^{\mathrm{j}}$, Roberto Cauda ${ }^{\mathrm{b}}$, \\ Claudio Viscoli ${ }^{\mathrm{c}}$, Russell Edward Lewis ${ }^{\text {a }}$, Pierluigi Viale ${ }^{\mathrm{a}}$, Mario Tumbarello ${ }^{\mathrm{b}}$ on behalf of \\ Italian Study Group on Resistant Infections of the Società Italiana Terapia Antinfettiva \\ (ISGRI-SITA)

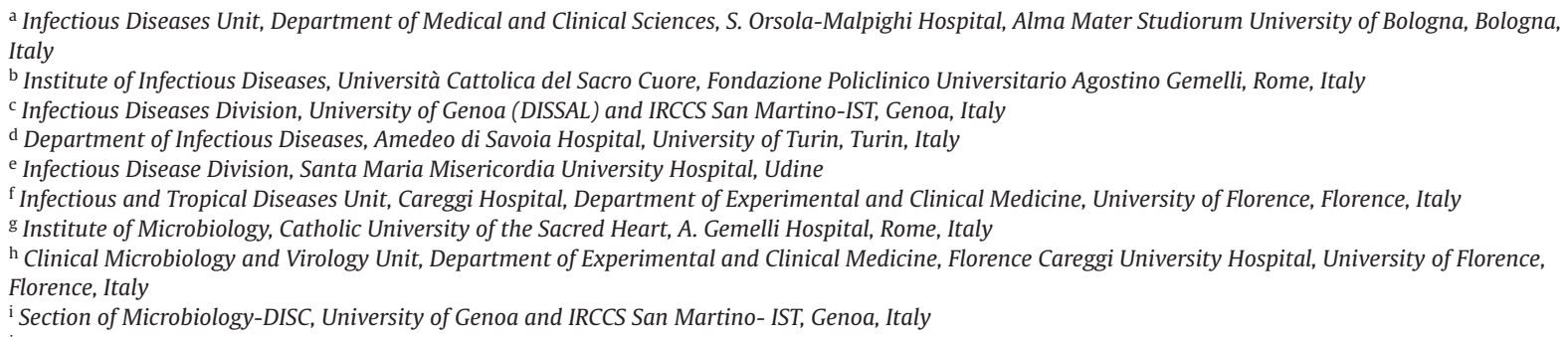

\section{A R T I C L E I N F O}

\section{Article history:}

Received 24 May 2017

Accepted 15 August 2017

Editor: J.-C. Lagier

\section{Keywords:}

Bloodstream infection

CR-KP

Combination therapy

Carbapenem

\begin{abstract}
A B S T R A C T
Objectives: To evaluate the impact of high-dose (HD) carbapenem-based combination therapy on clinical outcome in patients with monomicrobial carbapenem-resistant Klebsiella pneumoniae (CR-KP) bloodstream-infection (BSI).

Methods: Post hoc analysis of all adult patients with CR-KP BSI who were treated with a combination antibiotic regimen, collected over a six-year period in six large Italian teaching hospitals. To control for confounding effects of HD carbapenem combination on 14-day mortality, a multivariate Cox regression analysis was performed. Due to imbalances between patients, a propensity score for receiving HD carbapenem was added to the model.

Results: 595 patients with CR-KP BSI were analysed, $77 \%$ of isolates showed a carbapenem MIC $\geq 16 \mathrm{mg} /$ L, 428 (71.9\%) received HD carbapenem-based combination therapy. Overall, 127 patients (21.3\%) died within 14 days after BSI onset. Multivariate analysis showed the Charlson comorbidity index (HR 1.31, 95\%CI 1.20-1.43, $P<0.001$ ), septic shock at BSI onset (HR 3.14, 95\%CI 2.19-4.50, $P<0.001$ ), and colistinresistant strain (HR $1.52,95 \% \mathrm{CI} 1.02-2.24, P=0.03$ ) were independently associated with 14 -day mortality, whereas admission to surgical ward (HR 0.44, 95\%CI 0.25-0.78, $P=0.005$ ) and HD carbapenem use (HR $0.69,95 \% \mathrm{CI} 0.47-1.00, P=0.05$ ) were protective factors. When adjusted for the propensity score, HD carbapenem use showed a greater protective effect ( $\mathrm{HR} 0.64,95 \% \mathrm{CI} 0.43-0.95, P=0.03$ ). Stratifying the model for carbapenem MIC, the benefit of HD carbapenem was also observed for strains with carbapenem MIC $\geq 16 \mathrm{mg} / \mathrm{L}$.

Conclusions: In patients receiving combination therapy for CR-KP BSI, the use of HD carbapenem seems
\end{abstract} to be associated with better outcome, even in the presence of high-level carbapenem resistance.

(c) 2017 Elsevier B.V. and International Society of Chemotherapy. All rights reserved.

\footnotetext{
* Corresponding author. Infectious Diseases Unit, Department of Medical and Surgical Sciences, S. Orsola-Malpighi Hospital, Alma Mater Studiorum University of Bologna, Via Massarenti, 11, 40138, Bologna, Italy.

E-mail address: maddalena.giannella@libero.it (M. Giannella).
} 


\section{Introduction}

Over the last decade, the prevalence of carbapenem-resistant Klebsiella pneumoniae (CR-KP) infections has increased worldwide, with associated high morbidity and mortality, particularly among patients with bloodstream infection (BSI) [1].

Given the limited number of effective and safe agents, several strategies have been proposed to treat CR-KP infections. One of the most supported strategies is a combination antibiotic regimen to improve bactericidal activity, suppress the emergence of resistance, and overcome the pharmacokinetic weaknesses of individual agents. Indeed, several studies have reported lower mortality rates (0-40\%) among patients who received combination therapy compared with patients who received monotherapy (40-80\%) [2-7]. Recently, the positive impact of combination therapy has been shown to be significant, primarily in patients at high risk of dying [8].

Unfortunately, the question of which combination is superior remains unresolved [9]. Among the different combinations, those that included a carbapenem were associated with better outcome in some studies [4-7]. In an Italian multicentre study including 661 patients with CR-KP infection [7], the protective role of carbapenems was essentially confirmed for strains with MIC values $\leq 8 \mathrm{mg} / \mathrm{L}$, which represented around $30 \%$ of isolates in that cohort. On the other hand, carbapenem-sparing regimens are advocated by some authors for reducing carbapenem use in the context of infection control and antimicrobial stewardship programmes aimed to contain the high endemicity of CR-KP [10].

To assess the impact on 14-day mortality of a combination therapy with or without high-dose carbapenem in a cohort of CRKP BSI patients with high-level carbapenem resistance, we performed a post hoc analysis of the Italian cohort of patients with CR-KP infection, selecting those with BSI who received a combination therapy. A propensity score for receiving a carbapenem-containing combination was used to adjust the survival analysis.

\section{Material and methods}

\subsection{Study design, setting and population}

We performed a post hoc analysis of a multicentre, observational cohort study of all adult ( $\geq 18$ years) patients with CR-KP infection, hospitalised in six large tertiary-care teaching hospitals in Italy, from 01 January 2010 to 31 December 2015. Thus, with respect to the prior report [7], the current cohort includes patients hospitalised during a longer period (6 instead of 4 years) and in a larger number of centres ( 6 instead of 5 hospitals). Patients with monomicrobial CR-KP BSI who were treated with a combination regimen were selected for this study. Each patient was included only once at the time of the first positive blood cultures (BCs, index culture), even if more than one CR-KP BSI was reported.

\subsection{Definitions}

CR-KP BSI was defined as isolation of a CR-KP strain in the BCs obtained from a patient with clinical symptoms and/or signs of the systemic inflammatory response syndrome [4]. BSI onset was considered as the date of the index BCs collection (the first BCs yielding the study isolate). BSIs were classified as low-risk or high-risk depending on the source of the bacteraemia (urinary tract vs. all other identified and unidentified sources, respectively) [11]. BSIs were further considered as hospital-acquired, healthcare-associated or community-acquired, according to Friedman's criteria [12].

Septic shock was defined as sepsis associated with organ dysfunction and persistent hypotension despite volume replacement [13].
Combination therapy was defined as a regimen including two or more antibiotics, with at least one agent showing in vitro activity against the CR-KP isolate from BCs. Appropriate empirical antibiotic therapy was defined as treatment with at least one drug that had in vitro activity against the infecting organism, initiated within $48 \mathrm{~h}$ of the index BCs, and given in adequate dosage [14].

\subsection{Data}

Data were collected in a standardised case report form. Underlying diseases were recorded according to the Charlson comorbidity index. Invasive abdominal procedures included open abdominal surgery, endoscopic abdominal surgery and percutaneous drainage. Corticosteroid therapy was defined by $16 \mathrm{mg}$ prednisoneequivalent/day for $>15$ days; neutropenia was defined as $<500$ neutrophil cells $/ \mu \mathrm{L}$ of blood for $\geq 7$ days. Clinical severity at BSI onset was assessed according to APACHE III score and septic shock criteria [13]. Therapeutic variables included: administered antibiotics with their dosage, schedule, and duration. All cause 14-day mortality was collected for outcome. Patients were followed until hospital discharge or in-hospital mortality.

\subsection{Microbiological study}

Isolates were identified with the Vitek 2 system (bioMérieux, Marcy l'Etoile, France) and/or by matrix-assisted laser desorption ionization-time-of-flight (MALDI-TOF) mass spectrometry (MALDI Biotyper, Bruker Daltonics GmbH, Leipzig, Germany, or Vitek-MS, bioMérieux). The in vitro susceptibility of the isolates was assessed with the Vitek 2 system (bioMérieux) or the Sensititre broth microdilution method (Trek Diagnostic Systems, Cleveland, $\mathrm{OH}$ ). Results were interpreted in accordance with the European Committee on Antimicrobial Susceptibility Testing (EUCAST) clinical breakpoints.

\subsection{Statistical analysis}

Categorical variables were expressed as absolute numbers and their relative frequencies. Continuous variables were expressed as mean \pm standard deviation (SD) if normally distributed, or as median and interquartile range (IQR) if not normally distributed.

To analyse risk factors for 14-day mortality, non-survivors and survivors were compared. All the variables with a $P$-value $\leq 0.1$ at the univariate analysis were entered into a multivariate Cox backward regression model after assuming for proportional hazards. Patients were considered from the date of index BCs to death or until 14 days. Combination therapy with or without a carbapenem was introduced as the explanatory variable of interest. A propensity score for receiving carbapenem combination therapy was further added to the model.

The propensity score-the probability of receiving a carbapenem combination therapy-was calculated using a non-parsimonious multivariate logistic regression model in which the outcome variable was the use of carbapenem. The following variables were introduced into the model: age, sex, chronic renal failure, chronic liver failure, chemotherapy, corticosteroid treatment, abdominal invasive procedure, Charlson comorbidity index, healthcare-associated BSI, colistin-resistant strain, septic shock at BSI onset, carbapenem MIC, combination therapy containing gentamicin, and combination therapy containing tigecycline. The validity of the model was assessed by estimating goodness-of-fit to the data with the HosmerLemeshow test (69\%) and the ROC curve analysis with an areaunder-the-curve of 0.75 (95\%CI 0.71-0.79).

Statistical significance was considered for $P$ values $<0.05$. The software used for the analysis was SPSS (SPSS; version 21.0). 


\section{Results}

A total of 595 patients were analysed according to the study criteria. All strains were K. pneumoniae carbapenemase (KPC) producers, mostly KPC-3; $77 \%$ of them showed a carbapenem MIC $\geq 16 \mathrm{mg} / \mathrm{L}$.

Overall, 428 (71.9\%) patients received combination therapy containing a carbapenem. This consisted of meropenem administered at high doses ( $6 \mathrm{~g} /$ day) and by extended infusion (each infusion lasted $3 \mathrm{~h}$ ) in all the cases. The remaining $167(29.1 \%)$ patients received a combination therapy without a carbapenem. Comparison of the two groups is shown in Table 1.

A total of 127 (21.3\%) patients died within 14 days after BSI onset. Comparison of non-surviving and surviving patients showed significant differences for the Charlson comorbidity index (median 4 vs. $2, P<0.001$ ), APACHE III score at BSI onset (median 27 vs. 19 , $P<0.001$ ), underlying chronic renal failure (26\% vs. $15.4 \%, P=0.002$ ), admission to a surgical ward ( $10.2 \%$ vs. $22.4 \%, P=0.002)$, septic shock at BSI onset ( $40.9 \%$ vs. $12.8 \%, P<0.001)$, and colistin resistance $(27.6 \%$ vs $20.5 \%, P=0.09$ ) (see Table 2 ).

The multivariate Cox regression analysis showed that the Charlson comorbidity index (HR 1.31, 95\%CI 1.20-1.43, $P<0.001$ ), septic shock at BSI onset (HR 3.14, 95\%CI 2.19-4.50, $P<0.001$ ), and isolation of a colistin-resistant strain (HR 1.52, 95\%CI 1.02-2.24, $P<0.001$ ) were independently associated with 14-day mortality, whereas admis-

Table 1

Comparison of patients with carbapenem-resistant Klebsiella pneumoniae bloodstream infection who received combination therapy with and without high-dose carbapenem.

\begin{tabular}{|c|c|c|c|}
\hline & $\begin{array}{l}\text { Combination } \\
\text { with } \\
\text { carbapenem } \\
\mathrm{N}=428(\%)\end{array}$ & $\begin{array}{l}\text { Combination } \\
\text { without } \\
\text { carbapenem } \\
\mathrm{N}=167(\%)\end{array}$ & $P$ \\
\hline \multicolumn{4}{|l|}{ Demographic variables } \\
\hline Male sex & $269(62.9)$ & $97(58.1)$ & 0.30 \\
\hline Age (years) (median, IQR) & $66,54-76$ & $65,54-76$ & 0.67 \\
\hline \multicolumn{4}{|l|}{ Underlying conditions } \\
\hline Charlson score (median, IQR) & $3,2-6$ & $3,1-4$ & 0.007 \\
\hline APACHE III score (median, IQR) & $21,13-36$ & $19.5,15-38.5$ & 0.64 \\
\hline Chronic renal failure & $83(19.4)$ & $22(13.2)$ & 0.09 \\
\hline Haemodialysis & $59(13.8)$ & $14(8.4)$ & 0.09 \\
\hline Chronic liver disease & $46(10.7)$ & $8(4.8)$ & 0.02 \\
\hline Neutropenia & 55 (12.9) & $26(15.6)$ & 0.42 \\
\hline Corticosteroid therapy & $79(18.5)$ & $42(25.1)$ & 0.07 \\
\hline Chemotherapy & $59(13.8)$ & $35(21)$ & 0.03 \\
\hline Abdominal invasive procedures & $193(45.1)$ & $88(52.7)$ & 0.10 \\
\hline Transferred from an LTCF & $20(4.7)$ & $14(8.4)$ & 0.11 \\
\hline \multicolumn{4}{|l|}{ Admission ward at BSI onset } \\
\hline Medical ward & $176(41.1)$ & $70(41.9)$ & 0.93 \\
\hline Surgical ward & $78(18.2)$ & $40(24)$ & 0.13 \\
\hline Intensive care unit & $169(39.5)$ & $56(33.5)$ & 0.19 \\
\hline $\begin{array}{l}\text { Days of hospital stay before } \\
\text { BSI (median, IQR) }\end{array}$ & $50,29-77$ & $42,27.2-76.5$ & 0.19 \\
\hline \multicolumn{4}{|l|}{ Characteristics of BSI } \\
\hline Low-risk source & $87(20.3)$ & $38(22.8)$ & 0.57 \\
\hline High-risk source & $341(79.7)$ & $129(77.2)$ & 0.57 \\
\hline Septic shock & $71(16.6)$ & $41(24.6)$ & 0.02 \\
\hline Healthcare-associated & $28(6.5)$ & $20(12)$ & 0.03 \\
\hline Hospital-acquired & $385(90)$ & $146(87.4)$ & 0.37 \\
\hline \multicolumn{4}{|l|}{ Characteristics of the strain } \\
\hline Meropenem $\mathrm{MIC} \leq 8 \mathrm{mg} / \mathrm{L}$ & $103(24.1)$ & $64(38.3)$ & 0.001 \\
\hline Meropenem MIC $\geq 16$ mg/L & $325(75.9)$ & $103(61.7)$ & \\
\hline Colistin-resistant & $97(22.7)$ & $34(20.4)$ & 0.58 \\
\hline \multicolumn{4}{|l|}{ Therapeutic management } \\
\hline Colistin-containing regimen & $286(66.8)$ & $122(73.1)$ & 0.17 \\
\hline Tigecycline-containing regimen & $320(74.8)$ & $143(85.7)$ & 0.004 \\
\hline Gentamicin-containing regimen & $170(39.7)$ & $113(67.7)$ & $<0.001$ \\
\hline \multicolumn{4}{|l|}{ Outcome } \\
\hline 14-day mortality & 85 (19.9) & $42(25.1)$ & 0.18 \\
\hline In-hospital mortality & $122(28.5)$ & $54(32.3)$ & 0.36 \\
\hline
\end{tabular}

Abbreviations: BSI bloodstream infection; IQR interquartile range; LTCF long-term care facility.
Table 2

Comparison of patients with CR-KP BSI who died within 14 days after infection onset (date of positive index blood cultures) and survivors.

\begin{tabular}{|c|c|c|c|}
\hline & $\begin{array}{l}\text { Non-survivors } \\
\mathrm{N}=127(\%)\end{array}$ & $\begin{array}{l}\text { Survivors } \\
\mathrm{N}=468(\%)\end{array}$ & $P$ \\
\hline \multicolumn{4}{|l|}{ Demographic variables } \\
\hline Male sex & $77(60.6)$ & $289(61.8)$ & 0.84 \\
\hline Age (years) (median, IQR) & $66,57-77$ & $66,53-76$ & 0.18 \\
\hline \multicolumn{4}{|l|}{ Underlying conditions } \\
\hline Charlson score (median, IQR) & $4,3-6$ & $2,2-4$ & $<0.001$ \\
\hline APACHE III score (median, IQR) & $27,17-41$ & $19,13-35$ & $<0.001$ \\
\hline Chronic renal failure & $33(26)$ & $72(15.4)$ & 0.002 \\
\hline Haemodialysis & $20(15.7)$ & $53(11.3)$ & 0.22 \\
\hline Chronic liver disease & $16(12.6)$ & $38(8.1)$ & 0.16 \\
\hline Neutropenia & $17(13.4)$ & $64(13.7)$ & 1 \\
\hline Corticosteroid therapy & $34(26.8)$ & $87(18.6)$ & 0.05 \\
\hline Chemotherapy & $18(14.2)$ & $76(16.2)$ & 0.58 \\
\hline Abdominal invasive procedures & $60(47.2)$ & $221(47.2)$ & 1 \\
\hline Transferred from an LTCF & $10(7.9)$ & $24(5.1)$ & 0.27 \\
\hline \multicolumn{4}{|l|}{ Admission ward at BSI onset } \\
\hline Medical ward & $59(46.5)$ & $187(40)$ & 0.22 \\
\hline Surgical ward & $13(10.2)$ & $105(22.4)$ & 0.002 \\
\hline Intensive care unit & $55(43.2)$ & $170(36.3)$ & 0.18 \\
\hline \multicolumn{4}{|l|}{ Characteristics of BSI } \\
\hline Low-risk source & $25(19.7)$ & $100(21.4)$ & 0.71 \\
\hline High-risk source & $102(80.3)$ & $368(78.6)$ & \\
\hline Septic shock & $52(40.9)$ & $60(12.8)$ & $<0.001$ \\
\hline \multicolumn{4}{|l|}{ Characteristics of the strain } \\
\hline Meropenem MIC $\leq 8 \mathrm{mg} / \mathrm{L}$ & $32(25.2)$ & $135(28.8)$ & 0.44 \\
\hline Meropenem MIC $\geq 16 \mathrm{mg} / \mathrm{L}$ & $95(74.8)$ & $333(71.2)$ & \\
\hline Colistin-resistant & $35(27.6)$ & $96(20.5)$ & 0.09 \\
\hline \multicolumn{4}{|l|}{ Antibiotic management } \\
\hline Inadequate empirical treatment & $76(59.8)$ & $291(62.2)$ & 0.68 \\
\hline $\begin{array}{l}\text { Carbapenem-containing } \\
\text { combination }\end{array}$ & $85(66.9)$ & $343(73.3)$ & 0.18 \\
\hline Meropenem $\mathrm{MIC} \leq 8 \mathrm{mg} / \mathrm{L}$ & $15 / 85(17.6)$ & $88 / 343(25.7)$ & 0.15 \\
\hline Meropenem MIC $\geq 16 \mathrm{mg} / \mathrm{L}$ & $70 / 85(82.4)$ & $255 / 343(74.3)$ & 0.15 \\
\hline $\begin{array}{l}\text { Combination without } \\
\text { carbapenem }\end{array}$ & $42(33.1)$ & $125(26.7)$ & 0.18 \\
\hline 1 active drug & $28 / 42(66.7)$ & $90 / 125(72)$ & 0.56 \\
\hline$>1$ active drug & $14 / 42(33.3)$ & $35 / 127(28)$ & 0.56 \\
\hline
\end{tabular}

Abbreviations: BSI bloodstream infection; IQR interquartile range; LTCF long-term care facility.

sion to a surgical ward (HR 0.44, 95\%CI 0.25--0.78, $P=0.005$ ) was a protective factor. The carbapenem-containing combination (HR $0.69,95 \% 0.47-1.00, P=0.05)$ also remained in the final model as a protective factor, but with borderline significance (see appendix Table S1). When adjusted for the propensity score, the variables that remained in the model were the Charlson comorbidity index (HR $1.65,95 \%$ CI 1.10-2.46, $P<0.001$ ), corticosteroid therapy (HR 1.93 , $95 \%$ CI 1.22-3.04, $P=0.005$ ), admission to a surgical ward (HR 0.44 , $95 \% \mathrm{CI} 0.24-0.78, P=0.005$ ), septic shock at BSI onset (HR 4.70, 95\%CI $3.03-7.27, P<0.001$ ), colistin-resistant strain (HR 1.65, 95\%CI 1.10$2.46, P=0.005$ ), and carbapenem-containing combination (HR 0.64, $95 \%$ CI $0.43-0.95, P=0.03$ ) (appendix Table S1). Stratifying the model for the carbapenem MIC, the benefit of combination therapy with an HD carbapenem was also observed for strains with a carbapenem MIC $\geq 16$ mg/L (see Fig. 1).

\section{Discussion}

This is the first study that analyses the outcome of patients with CR-KP BSI treated with combination therapy, with or without a highdose meropenem, using the propensity score for accounting of population imbalances. Our results confirm previous data about the benefit of using a high-dose carbapenem as backbone in the combination regimens for CR-KP BSI.

The definition of combination therapy for carbapenem-resistant infection is a matter of debate [15]. Some experts define combination therapy as any regimen including more than one antibiotic with 


\section{$\mathrm{A}: \mathrm{MIC} \leq 8 \mathrm{mg} / \mathrm{L}$}

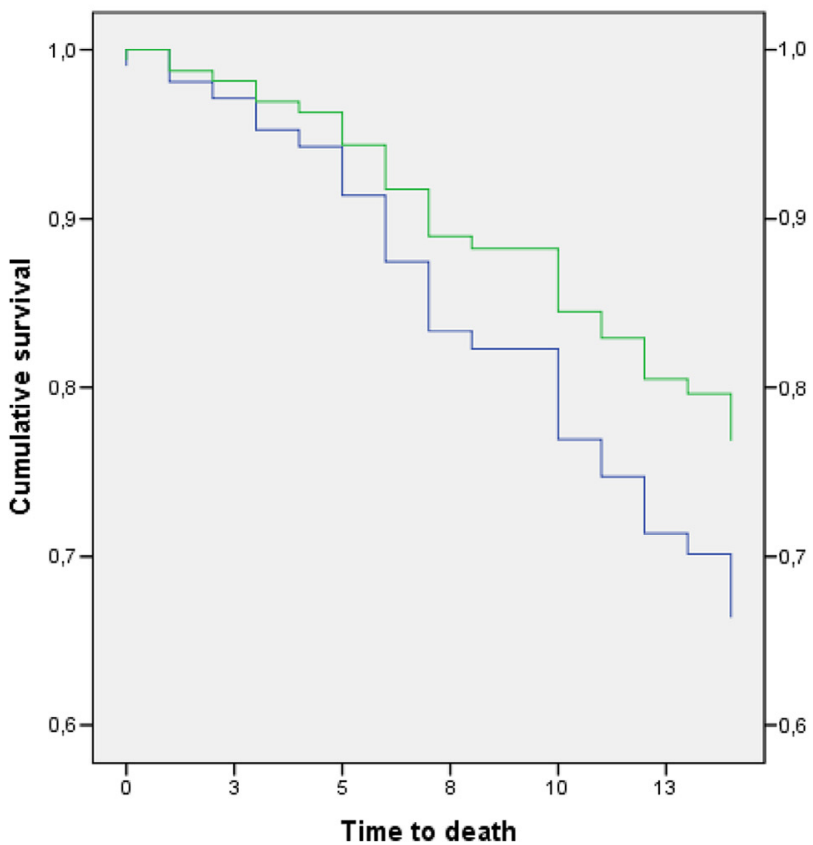

\section{$B: M I C \geq 16 \mathrm{mg} / \mathrm{L}$}
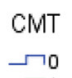

$\neg 1$

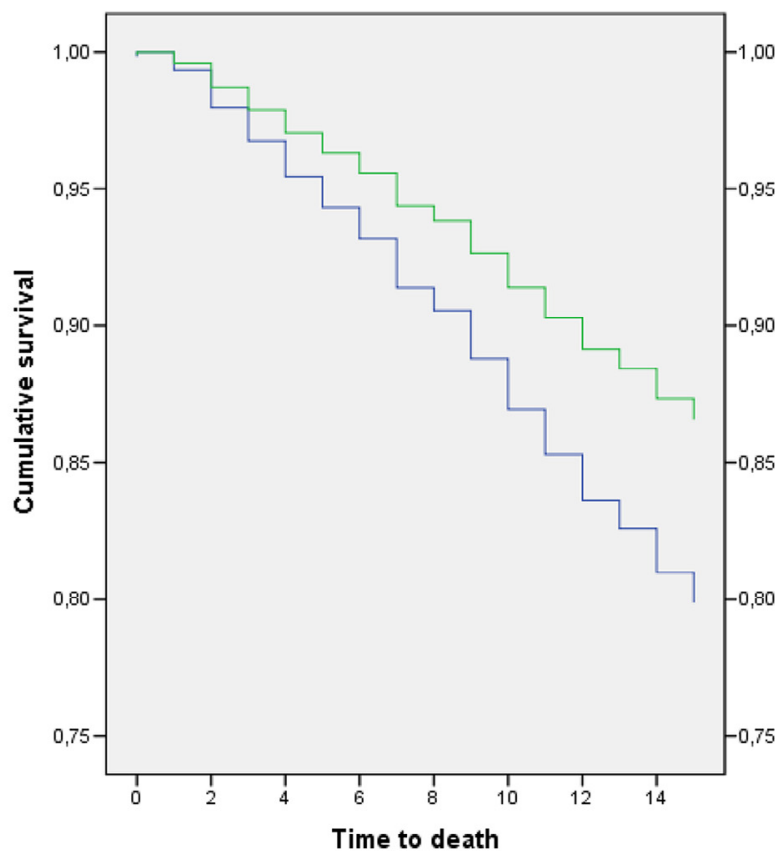

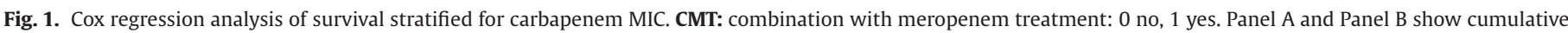

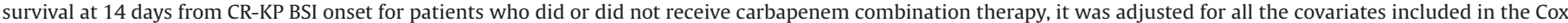

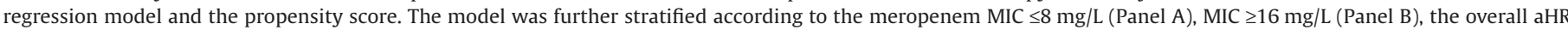
for the variable carbapenem combination therapy (CMT) was: $0.63,95 \% \mathrm{CI} 0.41-0.96, P=0.03$.

activity against Gram-negative bacteria (regardless of their in vitro activity against the infecting organism) [16], and others consider combination therapy as regimens including two or more in vitro active drugs [6]. As our objective was to assess the impact on outcome of meropenem use in carbapenem-resistant infections, we chose combinations with at least one in vitro active drug, because clinicians usually choose the meropenem companion based on in vitro susceptibility tests.

In a retrospective study of $141 \mathrm{CR}-\mathrm{KP}$ BSIs collected at two New York City hospitals from 2006-2013, the aim of the authors was to assess patient outcome according to the number of in vitro active drugs used, and whether an extended-spectrum $\beta$-lactam (meropenem or extended-spectrum cephalosporin) was administered [15]. Of the 111 isolates for which meropenem MICs were available, $90 \%$ had a meropenem MIC $\geq 16 \mathrm{mg} / \mathrm{L}$. A lower proportion of patients treated with meropenem died ( $24 \%$ vs. $37 \%$ ), but the difference was not statistically significant, including after adjusting for meropenem MIC ( $\leq 8 \mathrm{mg} / \mathrm{L}$ or $\geq 16 \mathrm{mg} / \mathrm{L}$ ) or meropenem dosing category (conventional, or high-dose administered by extended infusion). There was also no difference between single and multiple in vitro active drug used [15].

We reached different results by analysing a larger number of patients, investigating only the role of high-dose meropenem administered by extended infusion among patients treated with a combination regimen, and using the propensity score for accounting of possible imbalances. Indeed, in our univariate analysis, the 14-day mortality rate was not statistically significantly different between patients treated with and without HD meropenem (19.9\% vs. $25.1 \%, P=0.18$ ). However, the use of meropenem remained a protective factor in the multivariate model, and the level of significance increased after adjusting the analysis for the propensity score. Furthermore, we stratified our multivariate model for the meropenem MIC and observed a benefit of HD meropenem combination for strains with meropenem MIC $\geq 16 \mathrm{mg} / \mathrm{L}$, which represented $77 \%$ of overall strains. This could be explained by recent observation that high-dose/prolonged-infusion regimens of meropenem can reach the pharmacokinetic/pharmacodynamic target in patients with BSI caused by CR-KP with meropenem MICs up to 32-64 mg/L, but not for higher values $[17,18]$. Unfortunately, the lack of a punctual meropenem MIC in our strains with meropenem MIC $\geq 16 \mathrm{mg} / \mathrm{L}$ prevented us from establishing for which level of carbapenem resistance the use of carbapenems is still useful. Thus, the impact of carbapenem MIC on outcome in patients treated with carbapenems for CR-KP BSI should be further investigated.

Our study has some limitations. The study was not originally designed to analyse the impact of treatment on outcome, but to create an observational registry of severe infections with CR-KP. The adjustment of multivariate analysis for the most important confounding factors and for the propensity score should minimise this limitation. However, we acknowledge that the inclusion of propensity score still leads to residual confounding. Nevertheless, although there are some methodological drawbacks (retrospective observational study, lack of punctual meropenem MIC), the study has some important strengths: i) it is focused only on patients receiving carbapenem vs. non-carbapenem combination treatments; ii) it comprises a large number of patients with true CR-KP infection, as we selected only those with BSI; iii) the meropenem administration schedule was homogeneous (use of high doses by extended infusion); and iv) being an observational study, it reflects what happens in real life.

To conclude, in a population of patients receiving combination therapy for CR-KP BSI, with $77 \%$ of isolates showing a carbapenem MIC $\geq 16 \mathrm{mg} / \mathrm{L}$, the use of carbapenem seems to be associated with better outcome. Further studies, particularly clinical trials, should be performed to assess the level of carbapenem resistance for which the carbapenems are still useful.

Funding: No funding.

Competing interests: None. 
Ethical approval: Ethical approval was given by the IRB of the coordinator centre (Institute of Infectious Diseases, Catholic University of the Sacred Heart, A. Gemelli Hospital, Rome).

\section{Appendix A. Supplementary data}

Supplementary data associated with this article can be found, in the online version, at doi:10.1016/j.ijantimicag.2017.08.019.

\section{References}

[1] Capone A, Giannella M, Fortini D, Giordano A, Meledandri M, Ballardini M, et al. High rate of colistin resistance among patients with carbapenem-resistant Klebsiella pneumoniae infection accounts for an excess of mortality. Clin Microbiol Infect 2013;19:E23-30.

[2] Zarkotou O, Pournaras S, Tselioti P, Dragoumanos V, Pitiriga V, Ranellou K, et al. Predictors of mortality in patients with bloodstream infections caused by KPC-producing Klebsiella pneumoniae and impact of appropriate antimicrobial treatment. Clin Microbiol Infect 2011:17:1798-803.

[3] Qureshi ZA, Paterson DL, Potoski BA, Kilayko MC, Sandovsky G, Sordillo E, et al. Treatment outcome of bacteremia due to KPC-producing Klebsiella pneumoniae: superiority of combination antimicrobial regimens. Antimicrob Agents Chemother 2012;56:2108-13.

[4] Tumbarello M, Viale P, Viscoli C, Trecarichi EM, Tumietto F, Marchese A, et al. Predictors of mortality in bloodstream infections caused by Klebsiella pneumoniae carbapenemase-producing K. pneumoniae: importance of combination therapy. Clin Infect Dis 2012;55:943-50.

[5] Tzouvelekis LS, Markogiannakis A, Psichogiou M, Tassios PT, Daikos GL. Carbapenemases in Klebsiella pneumoniae and other Enterobacteriaceae: an evolving crisis of global dimensions. Clin Microbiol Rev 2012;25:682-707.

[6] Daikos GL, Tsaousi S, Tzouvelekis LS, Anyfantis I, Psichogiou M, Argyropoulou A, et al. Carbapenemase-producing Klebsiella pneumoniae bloodstream infections: lowering mortality by antibiotic combination schemes and the role of carbapenems. Antimicrob Agents Chemother 2014;58:2322-8.

[7] Tumbarello M, Trecarichi EM, De Rosa FG, Giannella M, Giacobbe DR, Bassetti $\mathrm{M}$, et al. Infections caused by KPC-producing Klebsiella pneumoniae: differences in therapy and mortality in a multicentre study. J Antimicrob Chemother 2015;70:2133-43.
[8] Gutierrez-Gutierrez B, Salamanca E, de Cueto M, Hsueh PR, Viale P, Pano-Pardo $J R$, et al. Effect of appropriate combination therapy on mortality of patients with bloodstream infections due to carbapenemase-producing Enterobacteriaceae (INCREMENT): a retrospective cohort study. Lancet Infect Dis 2017.

[9] Paul M, Carmeli Y, Durante-Mangoni E, Mouton JW, Tacconelli E, Theuretzbacher $\mathrm{U}$, et al. Combination therapy for carbapenem-resistant Gram-negative bacteria. J Antimicrob Chemother 2014;69:2305-9.

[10] Sbrana F, Malacarne P, Viaggi B, Costanzo S, Leonetti P, Leonildi A, et al. Carbapenem-sparing antibiotic regimens for infections caused by KPC-producing Klebsiella pneumoniae in Intensive Care Unit. Clin Infect Dis 2013;56:697-700.

[11] Rodriguez-Bano J, Picon E, Gijon P, Hernandez JR, Cisneros JM, Pena C, et al Risk factors and prognosis of nosocomial bloodstream infections caused by extended-spectrum-beta-lactamase-producing Escherichia coli. J Clin Microbiol 2010;48:1726-31.

[12] Friedman ND, Kaye KS, Stout JE, McGarry SA, Trivette SL, Briggs JP, et al. Health care-associated bloodstream infections in adults: a reason to change the accepted definition of community-acquired infections. Ann Intern Med 2002;137:791-7.

[13] Dellinger RP, Levy MM, Rhodes A, Annane D, Gerlach H, Opal SM, et al. Surviving sepsis campaign: international guidelines for management of severe sepsis and septic shock: 2012. Crit Care Med 2013;41:580-637.

[14] Daikos GL, Petrikkos P, Psichogiou M, Kosmidis C, Vryonis E, Skoutelis A, et al. Prospective observational study of the impact of VIM-1 metallo-beta-lactamase on the outcome of patients with Klebsiella pneumoniae bloodstream infections. Antimicrob Agents Chemother 2009;53:1868-73.

[15] Gomez-Simmonds A, Nelson B, Eiras DP, Loo A, Jenkins SG, Whittier S, et al. Combination regimens for treatment of carbapenem-resistant Klebsiella pneumoniae bloodstream infections. Antimicrob Agents Chemother 2016;60:3601-7.

[16] Gonzalez-Padilla M, Torre-Cisneros J, Rivera-Espinar F, Pontes-Moreno A Lopez-Cerero L, Pascual A, et al. Gentamicin therapy for sepsis due to carbapenem-resistant and colistin-resistant Klebsiella pneumoniae. J Antimicrob Chemother 2015;70:905-13.

[17] Del Bono V, Giacobbe DR, Marchese A, Parisini A, Fucile C, Coppo E, et al. Meropenem for treating KPC-producing Klebsiella pneumoniae bloodstream infections: should we get to the PK/PD root of the paradox? Virulence 2017;8:66-73.

[18] Pea F, Della Siega P, Cojutti P, Sartor A, Crapis M, Scarparo C, et al. Might real-time pharmacokinetic/pharmacodynamic optimisation of high-dose continuous-infusion meropenem improve clinical cure in infections caused by KPC-producing Klebsiella pneumoniae? Int J Antimicrob Agents 2017;49:255-8. 